\title{
Solitary fibrous tumor
}

\author{
Brian Davanzo $^{1}$, Robert E. Emerson ${ }^{2}$, Megan Lisy $^{1}$, Leonidas G. Koniaris ${ }^{1}$, Joshua K. Kays ${ }^{1}$ \\ ${ }^{1}$ Department of Surgery, Indiana University School of Medicine, Indianapolis, IN, USA; ${ }^{2}$ Department of Pathology and Laboratory Medicine, \\ Indiana University School of Medicine, Indianapolis, IN, USA \\ Contributions: (I) Conception and design: B Davanzo, LG Koniaris, JK Kays; (II) Administrative support: All authors; (III) Provision of study materials \\ or patients: All authors; (IV) Collection and assembly of data: All authors; (V) Data analysis and interpretation: All authors; (VI) Manuscript writing: \\ All authors; (VII) Final approval of manuscript: All authors. \\ Correspondence to: Joshua K. Kays. 545 Barnhill Drive, Emerson Hall 202, Indianapolis, IN, USA. Email: joshkays@iupui.edu.
}

\begin{abstract}
Solitary fibrous tumor (SFT) is a rare tumor of mesenchymal origin that account for less than $2 \%$ of all soft tissue masses. Initially identified in the pleura, SFT has been identified in multiple anatomic locations and can arise anywhere in the body. The varying histologic features along with non-specific means of identification have led SFT to be associated with several different names. Over the last several decades, sustained advances through research and technology have led to more reliable methods for differentiating this distinct soft tissue tumor. Advances specifically in immunohistochemistry and molecular diagnostics have identified CD34 as the most consistent marker in SFT, however even this lacks specificity to conclusively narrow down the broad differential for exact identification. More recently the discovery of the NAB2STAT6 fusion gene has led to more precise diagnosis of SFT. Like many other soft tissue tumors, surgical management is the mainstay of treatment for SFT with emphasis on obtaining tumor-negative margins. Radiation therapy and chemotherapy regimens have not demonstrated global effectiveness, and thus no standardized treatments have been identified. Given the rarity of SFT and current supportive evidence for therapies, management should be focused on tumor extirpation. Nonetheless, individualized therapy, determined within a multidisciplinary setting should be considered.
\end{abstract}

Keywords: Solitary fibrous tumors (SFT); sarcoma; hemangiopericytoma

Received: 07 November 2018; Accepted: 12 November 2018. Published: 21 November 2018.

doi: $10.21037 / \operatorname{tgh} .2018 .11 .02$

View this article at: http://dx.doi.org/10.21037/tgh.2018.11.02

\section{Introduction}

Solitary fibrous tumor (SFT) is a rare soft tissue tumor of mesenchymal origin. Originally described in pleural tumors by Klemperer and Rabin in 1931, these tumors have represented a unique diagnostic and treatment challenge (1). With gross and histologic features that overlap with many other soft tissue tumors, SFT have been assigned many different names in the past including benign mesothelioma, localized mesothelioma, solitary fibrous mesothelioma, and localized fibrous tumor to name a few. A prime example is the paper from Stout and Murray published only a decade after Klemperer and Rabin which described the histologic features of SFT but assigned the name hemangiopericytoma (2). More recently the discovery of the SFT fusion gene has led to the abandonment of the term hemangiopericytoma in favor of SFT. Due to the low incidence of SFT the majority of data has been derived from small retrospective series and case reports which has made developing specific diagnostic strategies and treatment planning difficult. Over the years, however, continued discovery and pooled research has led to further differentiation of hundreds of soft tissue masses including SFT. Furthermore advances in histologic, molecular and genetic techniques have allowed more precise categorization and identification of soft tissue tumors.

\section{Epidemiology}

While the etiology of SFT is currently unknown, data 

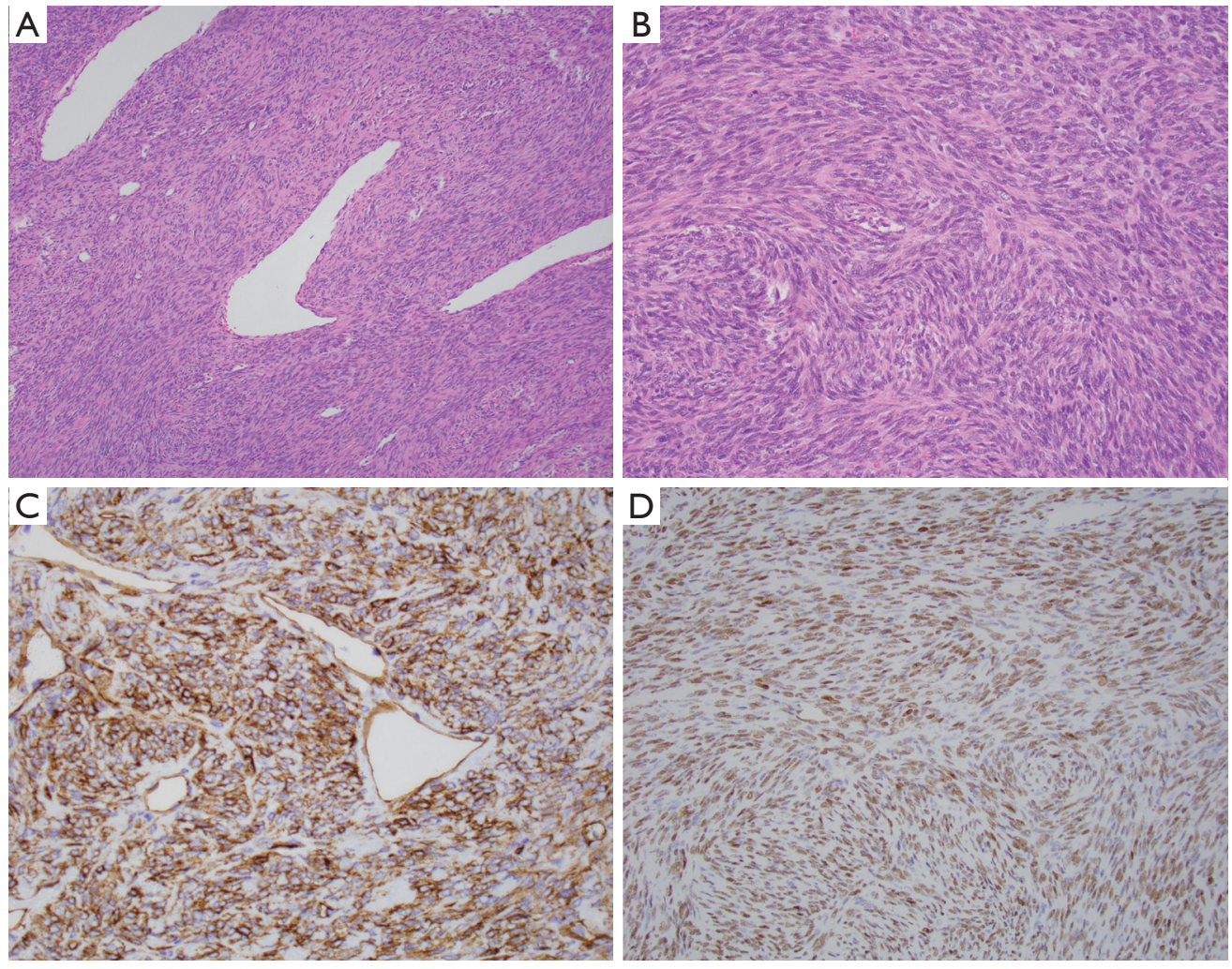

Figure 1 Solitary fibrous tumor involving the prostate with benign features. (A) Low power view with fascicular pattern and occasional large, sometimes branching blood vessels; (B) high power view showing oval to spindle-shaped nuclei and cells with focally fascicular pattern; (C) CD34 immunohistochemical stain with cytoplasmic staining; (D) STAT6 immunohistochemical staining with nuclear staining. H\&E staining; A: $\times 100 ;$ B,C,D: $\times 200$.

supports these tumors arise from cells of mesenchymal origin (3-5). It has an equal distribution between men and women $(6,7)$. SFTs may be diagnosed in adults of all ages, with most commonly presenting in the $5^{\text {th }}$ and $6^{\text {th }}$ decade (6-8). To date there are no environmental factors identified that increase the risk of SFT.

\section{Clinical presentation}

SFT tumors may be found in almost any site of the body with intra-thoracic being the most common location followed by intra-abdominal $(6,9,10)$. Figure 1 shows a detailed break down of tumor locations. Within the thoracic cavity the majority are pleural-based followed by lung parenchymal tumors, then the mediastinum and the diaphragm (6). They arise equally in the right and left chest (11). SFT in the mediastinum have a propensity to arise in the anterior mediastinum (12). The majority, between $50-80 \%$, of thoracic SFT will present as asymptomatic masses discovered incidentally on chest imaging $(6,11,13)$. Those that do present with symptoms present with nonspecific chest complaints such as chest pain, dyspnea or cough $(6,11,12,14)$.

The most common extrapleural site and second most common location overall is the abdomen (6). These have been noted to be in intra-peritoneal, retroperitoneal, and pelvic locations $(6,9,10,15)$. Intra-abdominal SFT are generally asymptomatic until they reach large enough size to cause mass effect on other organs.

Less common locations include the trunk, extremities, head and neck, and intracranial $(6,9,16,17)$. Those arising in the head and neck may arise from the sinonasal tract, oral cavity or the orbit (18-21). Intracranial SFT arise from the meninges (17). Tumors in these locations typically present with localized symptoms.

On rare occasions SFT can present with paraneoplastic syndromes, the most commonly described being non-islet cell hypoglycemia $(5,22)$. This is due to tumor production 
of high molecular weight insulin-like growth factor (IGF), specifically IGF-II (22). Seventy percent of these tumors will exhibit malignant behavior and presence of non-islet cell hypoglycemia is an overall poor prognostic indicator (22).

Tumor size at presentation is highly variable and is typically associated with location. The median size among several studies is noted between $7-10 \mathrm{~cm}$ with a range from anywhere from 1 to $40 \mathrm{~cm}(6,7,12,16)$. Grossly, these tumors are typically well circumscribed with smooth surfaces and many times a lobulated shape $(6,7)$.

\section{Work up/diagnosis}

The work up and diagnosis of SFT is analogous to many other soft tissue masses. Because many are asymptomatic or have non-specific symptoms, they are often found incidentally on imaging. Plain chest radiographs typically show a well-defined mass of variable size originating from the pleura with or without a pedicle (23). Contrast enhanced computed tomography (CT) will demonstrate a well-circumscribed, often lobulated, hypervascular tumor often with areas of necrosis especially when large $(4,24,25)$. T2-weighted magnetic resonance imaging (MRI) typically reveal a well-defined mass that is markedly inhomogeneous with large areas of bright signaling reflecting extensive areas of necrosis $(4,23)$.

A pre-treatment biopsy to diagnose and grade the mass is ideal. This biopsy should be performed by an individual experienced in soft tissue mass biopsy techniques. Open incisional biopsy by an experienced surgeon or, alternatively, core needle biopsy is preferred. Repeat biopsy can be considered if the initial biopsy is inadequate or fails to make the diagnosis. Depending on location, radiologic guided biopsy may prove to be helpful (26). The differential is typically vast and SFT is usually not high on this differential given the relative rarity. Classically, SFTs are composed of spindle to ovoid cells arranged in patternless distribution with areas of tumor cells and other hypocellular regions with higher percentage of stromal collagen $(6,15,27)$. Molecular genetic analysis has proven vital in further differentiating soft tissue masses for diagnosis. SFT has recently been associated with a NAB2-STAT6 gene fusion product that has proven to be highly specific and sensitive for SFT (28). This discovery of this gene fusion product has been pivotal for their diagnosis. However, the differential of a soft tissue mass with STAT6 expression should also include dedifferentiated liposarcoma (29). Further analysis and confirmation of positivity for MDM2 and CKD4 can help differentiate the two and confirm a diagnosis of dedifferentiated liposarcoma (15,30,31). Given the broad differential and genetic overlap in many of these tumors, work-up and management should be determined in a multidisciplinary setting, where personalized aspects of each individual case can be discussed and taken into account.

\section{Histopathology}

SFTs are variably cellular and composed of cells with oval to spindle-shaped nuclei with minimal cytoplasm and intervening collagen bands arranged in patternless distribution with areas highly rich in tumor cells while other areas are more hypocellular with higher percentage of stromal collagen (Figures $1 A, B, 2 A)(6,7,11,15,27)$. Nuclear atypia is usually minimal and mitotic figures are few in number, although exceptions occur $(6,7,11,15,27)$. Smaller tumors tend to be poorly vascularized with fewer mitoses and uniformed, elongated spindle cells and variable amounts of collagen versus larger tumors that have more pleomorphism and higher mitotic counts (Figure 2B,C) (4). Many cases may display a hemangiopericytic growth pattern due to pronounced vascularity, known as "staghorn" blood vessels, and perivascular sclerosis $(4,6,7,11,15,27)$.

Several unusual features that may suggest different diagnoses are occasionally seen and it should be recognized that these appearances do not exclude SFT. Fat production is seen in approximately $8 \%$ of tumors (32). Focal or extensive myxoid change or multinucleated giant cells also may be observed $(33,34)$.

CD34 has been noted to be one of the most consistent conventional markers in SFT and previously was the primary immunohistochemical (IHC) marker with expression in $79 \%$ of cases (Figure 1C) (35). Its lack of specificity, however, can occasionally be problematic $(6,7,21)$. Gastrointestinal stomal tumor, another CD34 positive stromal tumor that may occur in unexpected sites such as the retroperitoneum, should always be excluded through appropriate IHC staining for CD117/(c-KIT) and DOG1 (36). Many other markers are variably expressed in SFT such as vimentin, CD99, BCL2, nuclear $\beta$-catenin, and epithelial membrane antigen (EMA) $(6,15,16,37-39)$.

Strong nuclear STAT6 IHC staining has been used to reliably differentiate SFT from other soft tissue tumors, tumors of the head and neck, gynecologic tract and prostate (Figures 1D,2D) (40-43). In a recent review of $231 \mathrm{soft}$ 

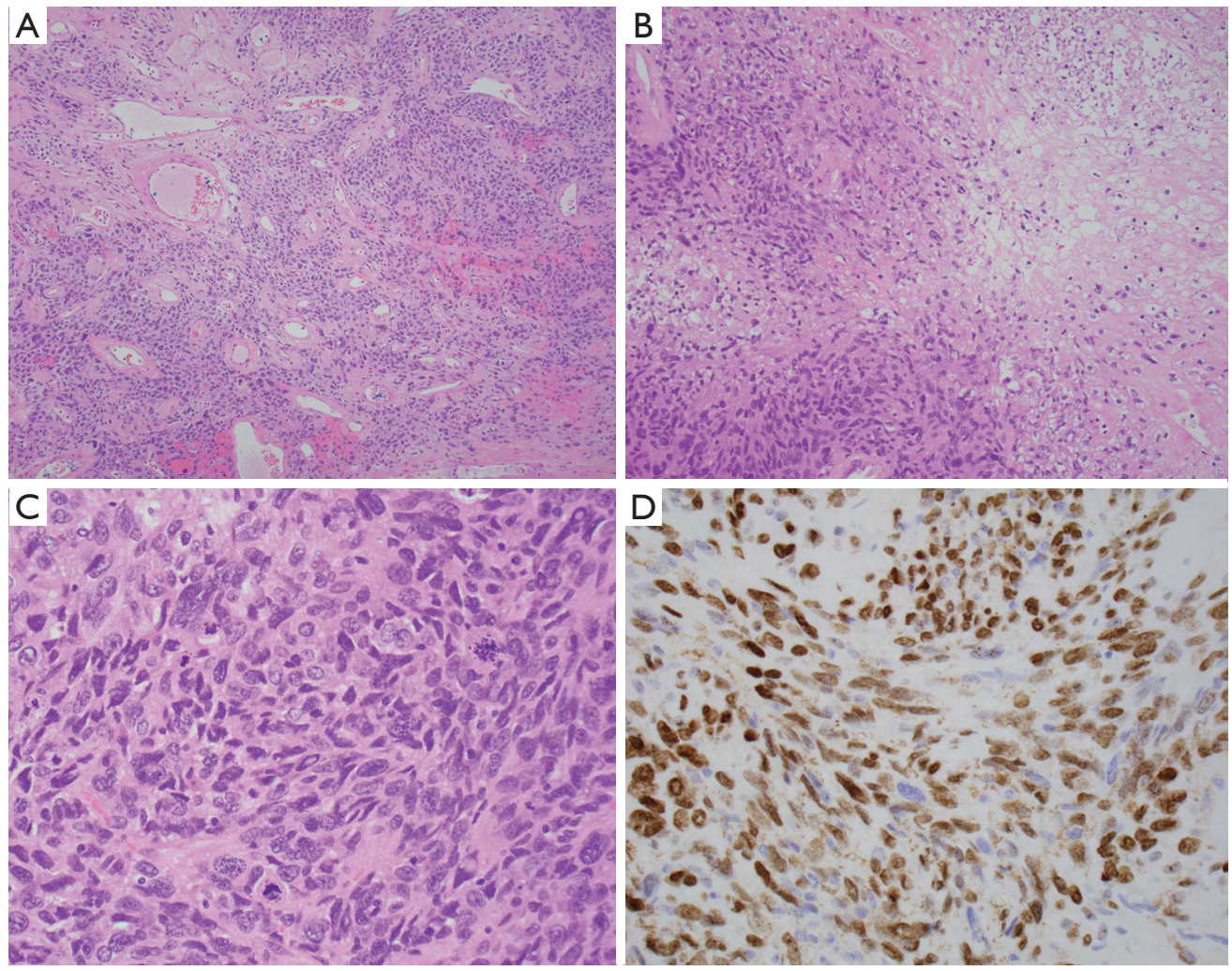

Figure 2 Solitary fibrous tumor of the pelvis of a male patient with malignant features. (A) Low power view with frequent, sometimes branching blood vessels; (B) high power view with nuclear enlargement and pleomorphism and coagulative tumor necrosis; (C) high power view showing overtly malignant nuclear features and frequent mitoses; (D) STAT6 immunohistochemical stain with nuclear staining. H\&E staining; A: $\times 100$; B,C,D: $\times 200$.

tissue tumors by Doyle et al., 59 of 60 (98\%) cases of SFT showed nuclear staining for STAT6 while all other tumor types were negative for STAT6 with the exception of 3 dedifferentiated liposarcomas (15). It should be noted that only strong, diffuse nuclear staining is highly specific for SFT, as weak staining can be seen in some other tumors.

Recently, multiple groups discovered a gene fusion product between $N A B 2$ and $S T A T 6$, which is a highly sensitive and specific marker for SFT and is considered the molecular hallmark of SFT. The STAT6-NAB2 fusion product results from an inversion at the $12 \mathrm{q} 13$ locus inducing expression of early growth response (EGR) mediated pathways (15). These fusions have been demonstrated by polymerase chain reaction to be present in $91 \%$ of SFT, with absence of this function in potentially histologically similar appearing tumors (44). This fusion may also be detected by a break-apart fluorescent in situ hybridization probe, seen in $58 \%$ of cases in one study (45). This did not, however, correlate with patient outcome.
Telomerase reverse transcriptase (TERT) promoter mutations have been seen in $28 \%$ of SFT and are associated with high risk pathologic characteristics and outcomes (46).

\section{Staging}

The 2013 World Health Organization classification of soft tissue sarcomas helped further differentiate many sarcomas with SFT being classified as a fibroblastic/myofibroblastic tumor (47). The American Joint Committee on Cancer (AJCC) staging system is the most commonly used staging system for soft tissue sarcomas. There are separate staging criteria depending on the location of each tumor which includes head and neck, trunk and extremities, abdomen and thoracic visceral organs, and retroperitoneum. Their system takes into account characteristics of the primary tumor $(\mathrm{T})$, lymph nodes $(\mathrm{N})$, distant metastasis $(\mathrm{M})$ and grade $(\mathrm{G})(48)$. The grade is determined by tumor differentiation, mitotic count and tumor necrosis (26). 


\section{Treatment}

Due to the paucity of randomized control trials there is no established, globally accepted treatment strategy. As a result, SFT should be approached in a manner similar to other soft tissue sarcomas, by a multidisciplinary team consisting of surgeons, medical oncologists, radiation oncologists, and additional ancillary support. This strategy has proven to improve outcomes in soft tissue sarcomas (49-52). For a more in depth overview on sarcoma management please see the article by Milgrom et al. in this special issue (53).

\section{Surgery}

Surgical management has been the mainstay treatment for SFTs $(4,6,7,11,13)$. Given the variable location of the primary tumor, surgical planning and intervention is highly variable based upon location and involved structures. Surgical management of SFTs is similar to most soft tissue sarcomas with a goal of wide resection margins and preservation of any critical surrounding organs or other structures. Obtaining adequate negative margins has been shown to decrease the rate local disease recurrence and improve survival (54). Several case series have demonstrated complete resection to be associated with low rates of local recurrence and progression to metastatic disease (4,9-13,55). Long-term follow up in small case series have demonstrated local recurrence as low as $8 \%$ after complete resection, however, true recurrence rates may be higher as recurrence has been reported to occur as far out as 17 years following resection $(4,56)$. Malignant SFT tumors, however, like other STS have a less durable response with up to $63 \%$ recurring even after complete resection (4). Thus, in instances of positive surgical margins, particularly in higher-grade SFT, surgical re-resection should be considered $(4,26)$.

\section{Radiation therapy}

The use of radiation therapy in treatment of SFTs is unfortunately limited by lack of data given the rarity of this tumor. Given the relatively good outcomes and indolent nature of this tumor, radiation therapy is not currently recommended after complete resection with negative margins. The indications and effectiveness of adjuvant radiation with positive margins or recurrent tumors has been debated but here too the benefit of radiation therapy remains undetermined. The role of adjuvant radiation has been most greatly studied in the CNS population (57). In an analysis of 155 patients with central nervous system SFTs, overall survival was noted to be higher $(93 \%$ vs. $88 \%)$ with surgery and radiation when compared to surgery alone, however this was not statistically significant $(\mathrm{P}=0.78)$ (57). This marginal potential benefit, however, has been corroborated in additional studies on intra-cranial SFT suggesting improved responses and outcomes with radiation therapy for patients with incomplete resection or metastatic disease $(58,59)$.

Although the role of adjuvant radiation for nonintracranial tumors is still unclear, small case series have suggested potential encouraging results. Kawamura et al. demonstrated a good response to radiation therapy in a 74-year old female with metastatic SFT of the pelvis (60). Saynak et al. have published their experience of treating a 66-year old patient with pleural SFT with radiation and achieving a significant response (61). Experiences such as these show the potential promise of radiation therapy for SFT, and support its consideration in a case-by-case basis likely as part of a multidisciplinary plan.

\section{Chemotherapy}

The relatively low incidence of SFTs has made it difficult to identify the most effective chemotherapy for advanced SFT. Further much of the data has been studied in a retrospective fashion. There have been multiple retrospective studies evaluating the effectiveness of standard cytotoxic chemotherapy with doxorubicin-based regimens. Results have been variable, but most have shown low or questionable response rates $(62,63)$. A study from the oncology group at Centre Leon Berard evaluated 23 patients who received first line cytotoxic chemotherapy. Only 2 partial responses were observed, 13 patients had stable disease and the remaining 8 patients' disease had progressed at 6 months (62). Because of these low response rates, more research has started looking into targeted therapies. In a retrospective analysis of 11 patients, combination therapy with temozolomide and bevacizumab was shown to have a partial response in $79 \%$, stable disease in $14 \%$, and progressive disease in 7\% (64). Additional research has looked into the sensitivity of multiple antiangiogenics including pazopanib, sorafenib, sunitinib, regorafenib, and axitinib (65). Sunitinib has been further studied and showed activity against SFT $(66,67)$. In a series of 31 patients, a partial response was seen in 2 patients, stabilization in 16 patients, and progression in 13 (66). As effectiveness of these chemotherapies is still 
Table 1 Tumor features associated with malignant behavior

\begin{tabular}{|c|c|}
\hline Study & Tumor features \\
\hline \multirow{7}{*}{$\begin{array}{l}\text { Gold } \\
\text { et al. (6) }\end{array}$} & Recurrent tumor \\
\hline & Macro- or microscopically positive resection margin \\
\hline & Size $>10 \mathrm{~cm}$ \\
\hline & $>4$ mitoses/10 HPFs \\
\hline & Increased nuclear pleomorphism \\
\hline & Increased cellularity \\
\hline & Presence of malignant components \\
\hline \multirow{4}{*}{$\begin{array}{l}\text { Demicco } \\
\text { et al. (7) }\end{array}$} & Age $\geq 55$ years \\
\hline & Size $\geq 15 \mathrm{~cm}$ \\
\hline & $\geq 4$ mitoses/10 HPFs \\
\hline & Tumor necrosis \\
\hline \multirow{5}{*}{$\begin{array}{l}\text { England } \\
\text { et al. (11) }\end{array}$} & Size $>10 \mathrm{~cm}$ \\
\hline & Tumor necrosis or hemorrhage \\
\hline & Increased cellularity \\
\hline & Nuclear pleomorphism \\
\hline & $>4$ mitoses/10 HPFs \\
\hline \multirow{4}{*}{$\begin{array}{l}\text { Wikin } \\
\text { et al. (12) }\end{array}$} & Size $\geq 10 \mathrm{~cm}$ \\
\hline & Hypercellularity \\
\hline & $>1$ mitosis/10 HPFs \\
\hline & Tumor necrosis or hemorrhage \\
\hline
\end{tabular}

HPFs, high power fields.

relatively poor, additional clinical studies are needed to better elucidate the molecular pathways involved with SFT in order to treat it more effectively.

\section{Prognosis}

While the behavior of most tumors is benign but potentially locally aggressive, a significant fraction of patients will have tumors that demonstrate malignant behavior. The biologic behavior of individual SFTs is hard to predict, and even tumors considered histologically benign may aggressively recur $(68,69)$. Nonetheless, overall the prognosis for SFT is better than many adenocarcinomas with 5 -year survival rates between $59-100 \%$ and 10 -year survival rates between $40 \%$ to $89 \%(4,54)$. In one of the larger reviews of SFT with analysis of 110 patients from MD Anderson in 2012, the overall 5 and 10-year disease specific survival was

(C) Translational Gastroenterology and Hepatology. All rights reserved. observed was $89 \%$ and $73 \%$ respectively (7).

Multiple factors have been associated with survival (Table 1). Gold et al. demonstrated recurrent tumors, macro- or microscopically positive resection margins, tumor size $>10 \mathrm{~cm},>4$ mitoses $/ 10 \mathrm{HPF}$, increased nuclear pleomorphism, increased cellularity and presence of malignant component all to be poor prognostic factors (6). In another large study by England et al. they identified size $>10 \mathrm{~cm}$, tumor necrosis or hemorrhage, increased cellularity, pleomorphism, and $>4$ mitoses/10 HPF to be consistent with malignant behavior and worse prognosis (11). A smaller series published by Witkin et al. found similar findings to both Gold and England (12). Demicco et al. developed and proposed a risk stratification model to classify patients into low, intermediate and high risk based on patient age, tumor size and mitotic figures/10 HPF (7).

\section{Surveillance}

Routine follow up is essential for SFT after surgical resection (54). Oncologic surveillance provides opportunity for early treatment of recurrent or metastatic disease. Given the low incidence of SFT, there are not currently agreed upon guidelines for surveillance. Many research groups have attempted to create risk models in order to guide post-operative surveillance with varying results $(6,7)$. As stated above, the review from MD Anderson displayed that tumors greater than $15 \mathrm{~cm}$ in older patients (greater than 55) with mitotic figures greater than or equal to 4/10 high power fields were at high risk of metastasis deserving close surveillance (7). Surveillance of each patient should be patient specific dependent on tumor location and other patient factors. While many oncologic diseases are followed for limited periods of times such as 5 years, SFT has been diagnosed in many patients later in life potentiating the need for lifetime surveillance as recurrence has been noted to occur many years after initial diagnosis and treatment (56).

\section{Future perspectives}

Given the rarity of SFT and mostly retrospective data from small cases series, there is still much progress to be made. Although researchers have gained significant understanding of the molecular pathways of SFT, the effectiveness of most chemotherapies against SFT is relatively poor. Additional clinical studies are needed to better elucidate the molecular pathways involved with SFT in order to identify more 
efficient targeted therapy. This will further lead to more personalized analysis of each patient's tumor's biology leading to better ways to assess prognosis and treat each individual tumor. In a study, out of Human Pathology in 2014, researchers evaluated the variations of the NAB2STAT6 fusion gene and were able to differentiate which mutations were more likely to be aggressive than others (70).

Further, some researchers have begun analyzing the effectiveness of chemotherapy in regard to degree of differentiation and aggressiveness of the primary tumor (67). Stacchiotti interestingly found that more aggressive SFTs seemed more sensitive to cytotoxic chemotherapy (65-67).

\section{Conclusions}

SFTs are a rare slow-growing mesenchymal tumor that has been differentiated from other soft tissue tumors over the past decades through advances in histology and molecular genetics. While surgical resection is the mainstay of treatment and provides favorable outcomes for resectable disease, radiation and chemotherapy may have potential roles in the treatment algorithm. Optimal treatment of advanced and metastatic disease is still unclear, but currently involves a combination of surgical, radiation, and chemotherapeutic strategies under the supervision of a multidisciplinary team. Increasing research into various chemotherapy regimens and radiation has shown encouraging results, but more research is needed for further clinical translation.

\section{Acknowledgements}

None.

\section{Footnote}

Conflicts of Interest: The authors have no conflicts of interest to declare.

\section{References}

1. Klemperer P, Rabin CB. Primary neoplasms of the pleura. A report of five cases. Arch Pathol 1931;11:385-412.

2. Stout AP, Murray MR. Hemangiopericytoma. A vascular tumor featuring Zimmerman's pericytes. Ann Surg 1942;116:26-33.

3. Chan JK. Solitary fibrous tumour: everywhere and a diagnosis in vogue. Histopathology 1997;31:568-76.

4. Robinson LA. Solitary fibrous tumor of the pleura. Cancer
Control 2006;13:264-9.

5. Bruzzone A, Varaldo M, Ferrarazzo C, et al. Solitary fibrous tumor. Rare Tumors 2010;2:e64.

6. Gold JS, Antonescu CR, Hajdu C, et al. Clinicopathologic correlates of solitary fibrous tumors. Cancer 2002;94:1057-68.

7. Demicco EG, Park MS, Araujo DM, et al. Solitary fibrous tumor: a clinicopathological study of 110 cases and proposed risk assessment model. Mod Pathol 2012;25:1298-306.

8. Cardillo G, Facciolo F, Cavazzana A, et al. Localized (solitary) fibrous tumors of the pleura: an analysis of 55 patients. Ann Thorac Surg 2000;70:1808-12.

9. Hasegawa T, Matsuno Y, Shimoda T, et al. Extrathoracic solitary fibrous tumors: Their histological variability and potentially aggressive behavior. Hum Pathol 1999;30:1464-73.

10. Morimitsu Y, Nakajima M, Hisaoka M, et al. Extrapleural solitary fibrous tumor: clinicopathologic study of 17 cases and molecular analysis of the p53 pathway. APMIS 2000;108:617-25.

11. England DM, Hochholzer L, McCarthy MJ. Localized benign and malignant fibrous tumors of the pleura. A clinicopathologic review of 223 cases. Am J Surg Pathol 1989;13:640-58.

12. Witkin GB, Rosai J. Solitary fibrous tumors of the mediastinum. A report of 14 cases. Am J Surg Pathol 1989;13:547-57.

13. Sung SH, Chang JW, Kim J, et al. Solitary fibrous tumors of the pleura: surgical outcome and clinical course. Ann Thorac Surg 2005;79:303-7.

14. de Perrott M, Kurt AM, Robert JH, et al. Clinical behavior of solitary fibrous tumors of the pleura. Ann Thorac Surg 1999;67:1456-9.

15. Doyle LA, Vivero M, Fletcher CDM, et al. Nuclear expression of STAT6 distinguishes solitary fibrous tumor from histologic mimics. Mod Pathol 2014;27:390-5.

16. Brunnemann RB, Ro JY, Ordonez NG, et al. Extrapleural solitary fibrous tumors: a clinicopathologic study of 24 cases. Mod Pathol 1999;12:1034-42.

17. Carneiro SS, Scheithauer BW, Nascimento AG, et al. Solitary fibrous tumor of the meninges: a lesion distinct from fibrous meningioma: a clinicopathologic and immunohistochemical study. Am J Clin Pathol 1996;106:217-24.

18. Dorfman DM, To K, Dickersin GR, et al. Solitary fibrous tumor of the orbit. Am J Surg Pathol 1994;18:281-7.

19. DeBacker CM, Bodker F, Putterman AM, et al. Solitary 
fibrous tumor of the orbit. Am J Ophthal 1996;121:447-9.

20. Ing EB, Kennerdell JS, Olson PR, et al. Solitary fibrous tumor of the orbit. Ophthalmic Plast Reconstr Surg 1998;14:57-61.

21. Westra WH, Gerald WL, Rosai J. Solitary fibrous tumor. Consistent CD34 immunoreactivity and occurrence in the orbit. Am J Surg Pathol 1994. 18(10):992-8.

22. Herrmann BL, Saller B, Kiess W, et al. Primary malignant histiocytoma of the lung: IGF-II producing tumor induces fasting hypoglycemia. Exp Clin Endocrinol Diabetes 2000;108:515-8.

23. Ali SZ, Hoon V, Hoda S, et al. Solitary fibrous tumor. A cytologic-histologic study with clinical, radiologic, and immunohistochemical correlations. Cancer 1997;81:116-21.

24. Dedrick CG, McLoud TC, Shepard JA, et al. Computed tomography of localized pleural mesothelioma. AJR Am J Roentgenol 1985;144:275-80.

25. Lee KS, Im JG, Choe KO, et al. CT findings in benign fibrous mesothelioma of the pleura: pathologic correlation in nine patients. AJR Am J Roentgenol 1992;158:983-6.

26. National Comprehensive Cancer Network. Soft Tissue Sarcoma (V 2.2018). Available online: https://www.nccn. org/professionals/physician/gls/pds/sarcoma.pdf. Accessed 2018 October 18.

27. Yokoi T, Yatabe Y, Suzuki M, et al. Solitary fibrous tumors: significance of p53 and CD34 immunoreactivity in its malignant transformation. Histopathology 1998;32:423-32.

28. Robinson DR, Wu YM, Sundaram SK, et al. Identification of recurrent NAB2-STAT6 gene fusions in solitary fibrous tumor by integrative sequencing. Nat Genet 2013;45:180-5.

29. Doyle LA, Tao D, Enriquez AM. STAT6 is amplified in a subset of dedifferentiated liposarcoma. Mod Pathol 2014;27:1231-7.

30. Creytens D, Libbrecht L, Ferdinande L. Nuclear expression of STAT6 in dedifferentiated liposarcoma with solitary fibrous tumor-like morphology: a diagnostic pitfall. Appl Immunohistochem Mol Morphol 2015;23:462-3.

31. Italiano A, Bianchini L, Keslair F, et al. HMGA2 is the partner of MDM2 in well-differentiated and dedifferentiated liposarcoma whereas CDK4 belongs to distinct inconsistent amplicon. Int J Cancer 2008;122:2233-41.

32. Smith SC, Gooding WE, Elkins M, et al. Solitary fibrous tumors of the head and neck: A multi-institutional clinicopathologic study. Am J Surg Pathol 2017;41:1642-56.

33. Lau SK, Weiss LM, Chu PG. Myxoid solitary fibrous tumor: a clinicopathologic study of three cases. Virchows
Arch 2009;454:189-94.

34. Yuzawa S, Tanikawa S, Kunibe I, et al. A case of giant cellrich solitary fibrous tumor in the external auditory canal. Pathol Int 2016;66:701-5.

35. Flint A, Weiss SW. CD-34 and keratin expression distinguishes solitary fibrous tumor (fibrous mesothelioma) of pleura from desmoplastic mesothelioma. Hum Pathol 1995;26:428-31.

36. Miettinen M, Felisiak-Golabek A, Wang Z, et al. GIST manifesting as a retroperitoneal tumor: clinicopathologic immunohistochemical, and molecular genetic study of 112 cases. Am J Surg Pathol 2017;41:577-85.

37. Bhattacharya, B, Dilworth HP, Donahue, et al. Nuclear $\beta$-catenin expression distinguishes deep fibromatosis from other benign and malignant fibroblastic and myofibroblastic lesions. Am J Surg Pathol 2005;29:653-9.

38. Carlson JW, Fletcher CDM. Immunohistochemistry for $\beta$-catenin in the differential diagnosis of spindle cell lesions: analysis of a series and review of the literature. Histopathology 2007;51:509-14.

39. Magro G, Bisceglia M, Michal M, et al. Spindle cell lipoma-like tumor, solitary fibrous tumor and myofibroblastoma of the breast: a clinic-pathologic analysis of 13 cases in favor of underlying histogenic concept. Virchows Archiv 2002;440:249-60.

40. Cheah AL, Billings SD, Goldblum JR, et al. STAT6 rabbit monoclonal antibody is a robust diagnostic tool for the distinction of solitary fibrous tumour from its mimics. Pathology 2014;46:389-95.

41. Smith MH, Islam NM. STAT6 reliably distinguishes solitary fibrous tumors from myofibromas. Head Neck Pathol 2018;12:110-7.

42. Yang EJ, Howitt BE, Fletcher CDM, et al. Solitary fibrous tumour of the female genital tract: a clinicopathological analysis of 25 cases. Histopathology 2018;72:749-59.

43. Guner G, Bishop JA, Bezerra SM, et al. The utility of STAT6 and ALDH1 expression in the differential diagnosis of solitary fibrous tumor versus prostate-specific stromal neoplasms. Hum Pathol 2016;54:184-8.

44. Rekhi B, Shetty O, Tripathi P, et al. Molecular characterization of a series of solitary fibrous tumors, including immunohistochemical expression of STAT6 and NATB2-STAT6 fusion transcripts, using reverse transcriptase (RT)-polymerase chain reaction (PCR) technique: an Indian experience. Pathol Res Pract 2017;213:1404-11.

45. Kouba E, Simper NB, Chen S, et al. Solitary fibrous tumour of the genitourinary tract: a clinicopathologic 
study of 11 cases and their association with the NAB2STAT6 fusion gene. J Clin Pathol 2017;70:508-14.

46. Bahrami A, Lee S, Schaefer IM, et al. TERT promoter mutations and prognosis in solitary fibrous tumor. Mod Pathol 2016;29:1511-22.

47. Doyle LA. Sarcoma classification: an update based on the 2013 World Health Organization classification of tumors of soft tissue and bone. Cancer 2014;120:1763-74.

48. Amin MD, Edge S, Greene F, et al. AJCC Cancer Staging Manual. 8th ed. Cham. Switzerland; Springer International Publishing: 2017.

49. Gutierrez JC, Perez EA, Moffat FL, et al. Should soft tissue sarcomas be treated at high-volume centers? An analysis of 4205 patients. Ann Surg 2007;245:952.

50. Gutierrez JC, Perez EA, Franceschi D, et al. Outcomes for soft-tissue sarcoma in 8249 cases from a large state cancer registry. JSR 2007;141:105-14.

51. Perez EA, Gutierrez JC, Moffat FL, et al. Retroperitoneal and truncal sarcomas: prognosis depends upon type not location. Ann Surg Oncol 2007;14:1114-22.

52. Jawad MU, Cheung MC, Min ES, et al. Ewing sarcoma demonstrates racial disparities in incidence-related and sex-related differences in outcome. An analysis of 1631 cases from the SEER database, 1973-2005. Cancer 2009; 115:3526-36.

53. Milgrom DP, Sehdev A, Kays JK, et al. Integrating therapies for surgical adult soft tissue sarcoma patients. Transl Gastroenterol Hepatol 2018;3:88.

54. Kayani B, Sharma A, Sewell MD, et al. A review of the surgical management of extrathoracic solitary fibrous tumors. Am J Clin Oncol 2018;41:687-94.

55. Briselli M, Mark EJ, Dickersin GR. Solitary fibrous tumors of the pleura: eight new cases and review of 360 cases in the literature. Cancer 1981;47:2678-89.

56. Okike N, Bernatz PE, Wooner LB. Localized mesothelioma of the pleura: benign and malignant variants. J Thorac Cardiovasc Surg 1978;75:363-72.

57. Rana N, Kim E, Jaboin J, et al. The role of adjuvant radiation in the management of solitary fibrous tumors of the central nervous system: a National Cancer Database analysis of 155 patients. Cureus 2018;10:e2656.

58. Stessin AM, Sison C, Nieto J, et al. The role of postoperative radiation therapy in the treatment of meningeal hemangiopericytoma - experience from the SEER database. Int J Radiat Oncol Biol Phys 2013;85:784-90.

59. Haas RL, Walrayen I, Lecointe-Artzner E, et al. Radiation therapy as the sole management for solitary fibrous tumors (SFT): a retrospective study from the Global SFT Initiative in collaboration with the Sarcoma Patients EuroNet. Int J Radiat Oncol Biol Phys 2018;101:1226-33.

60. Kawamura S, Nakamura T, Ova T, et al. Advanced malignant solitary fibrous tumor in pelvis responding to radiation therapy. Pathol Int 2007;57:213-8.

61. Saynak M, Bayir-Angin G, Kocak Z, et al. Recurrent solitary fibrous tumor of the pleura: significant response to radiotherapy. Med Oncol 2010;27:45-8.

62. Levard A, Derbel O, Meeus P, et al. Outcome of patients with advanced solitary fibrous tumors: the Centre Leon Berard experience. BMC Cancer 2013;13:109.

63. Park MS, Ravi V, Conley A, et al. The role of chemotherapy in advanced solitary fibrous tumors: a retrospective analysis. Clin Sarcoma Res 2013;3:7.

64. Park MS, Patel SR, Ludwig JA, et al. Activity of temozolomide and bevacizumab in the treatment of locally advanced, recurrent, and metastatic hemagniopericytoma and malignant solitary fibrous tumors. Cancer 2011;117:4939-47.

65. Stacchiotti S, Tortoreto M, Baldi GG, et al. Preclinical and clinical evidence of activity of pazopanib in solitary fibrous tumour. Eur J Cancer 2014;50:3021-8.

66. Stacchiotti S, Negri T, Libertini M, et al. Sunitinib malate in solitary fibrous tumor (SFT). Ann Oncol 2012;23:3171-79.

67. Stacchiotti S, Libertini M, Negri T, et al. Response to chemotherapy of solitary fibrous tumour: a retrospective study. Eur J Cancer 2013;49:2376-83.

68. Gholami S, Cassidy MR, Kirane A, et al. Size and location are the most important risk factors for malignant behavior in resected solitary fibrous tumors. Ann Surg Oncol 2017;24:3865-71.

69. Vallat-Decouvelaere AV, Dry SM, Fletcher CD. Atypical and malignant solitary fibrous tumors in extrathoracic locations: evidence of their comparability to intra-thoracic tumors. Am J Surg Pathol 1998;22:1501-11.

70. Akaike K, Kurisaki-Arakawa A, Hara K, et al. Distinct clinicopathologic features of NAB2-STAT6 fusion gene variants in solitary fibrous tumor with emphasis on the acquisition of highly malignant potential. Hum Pathol 2015;46:347-56.

doi: $10.21037 / \operatorname{tgh} .2018 .11 .02$

Cite this article as: Davanzo B, Emerson RE, Lisy M, Koniaris LG, Kays JK. Solitary fibrous tumor. Transl Gastroenterol Hepatol 2018;3:94. 